\title{
Reducing Pain Experienced During Potassium Chloride Infusion in the Emergency Department
}

\author{
Jen Heng Pek', Hui Cheng Tan'1, Puneet Seth', Evelyn Wong² \\ 'Sengkang Health, Singhealth, Singapore \\ ${ }^{2}$ Singapore General Hospital, Singhealth, Singapore
}

Cite this article as: Pek JH, Tan HC, Seth P, Wong E. Reducing Pain Experienced During Potassium Chloride Infusion in the Emergency Department. Eurasian J Emerg Med 2017; 16: 148-52

\begin{abstract}
Aim: Patients with hypokalemia often complained of pain during intravenous potassium chloride infusion. By administering lignocaine prior to the infusion, the pain experienced may be reduced but safety concerns have limited its clinical application. The primary objective was to determine the efficacy of lignocaine in reducing pain experienced during potassium chloride infusion in the Emergency Department. The secondary objective was to determine the adverse events associated with intravenous lignocaine use for this purpose.

Materials and Methods: Continuous quality improvement data were prospectively collected. The lignocaine protocol involved giving $30 \mathrm{mg}$ of $1 \%$ lignocaine intravenously 1 minute before starting the potassium chloride infusion. The decision to use this protocol was left to the discretion of the attending doctor when managing a patient with hypokalemia requiring intravenous replacement. The nurse taking care of the patient would record any experience of pain during the infusion. Occurrence of any adverse event was recorded.

Results: A total of 100 patients were recruited. After stabilized weights adjustment, the resultant sample size was 98 , with 49 patients in each group. The proportion of patients experiencing pain was significantly higher when no lignocaine was given ( $38.8 \% \mathrm{vs} 16.3 \%, \mathrm{p}=0.013)$. The absolute risk reduction was $22.5 \%$, and the relative risk of pain was $0.42(0.20-0.87, \mathrm{p}=0.013)$. The numbers needed to treat was five (range, $3-19)$. There was no adverse event associated with the lignocaine protocol in this study.
\end{abstract}

Conclusion: Intravenous lignocaine was efficacious and safe in reducing pain during potassium chloride infusion.

Keywords: Lignocaine, pain, potassium chloride, safe

\section{Introduction}

Hypokalemia is a common electrolyte abnormality encountered in the clinical setting. It can lead to cardiac arrhythmias, muscle weakness, and rhabdomyolysis (1-5). The causes of hypokalemia can be broadly classified into decreased intake, increased entry into cells due to causes such as increase in extracellular $\mathrm{pH}$, increased $\beta$-adrenergic activity or insulin administration, and increased losses from sweating, and the gastrointestinal or renal systems (6). In addition to the diagnosis and treatment of the underlying cause(s) of hypokalemia, replacement of the potassium deficit is necessary when managing these patients. This urgency of replacement therapy will depend on the severity of hypokalemia, the rate of decline in serum potassium concentration, and associated comorbidities of the patient. Potassium can be administered as potassium chloride, potassium phosphate, potassium bicarbonate potassium gluconate or its precursors (potassium citrate, potassium acetate) $(7,8)$. Potassium chloride has been the mainstay of replacement therapy for two main reasons:

- The serum potassium concentration increases at a faster rate than other formulation as chloride is primarily an extracellular 
anion, thereby promoting maintenance of the administered potassium in the extracellular fluid (9).

- Patients with hypokalemia are often chloride-depleted. This chloride-depleted state will contribute to metabolic alkalosis by enhancing renal bicarbonate reabsorption that leads to potassium wasting, as sodium is reabsorbed in exchange for secreted potassium rather than with chloride $(10,11)$.

Intravenous replacement should be used in patients with severe symptomatic hypokalemia (less than $2.5 \mathrm{mmol} / \mathrm{L}$ ) and in patients with less severe hypokalemia who are unable to take oral medications. The most common problem associated with potassium chloride infusion is pain. The occurrence of pain has been reported in up to $60 \%$ of patients receiving intravenous replacement (12), resulting in patient's refusal of the prescribed therapy (13). While the exact mechanism of action is not known, possible causes include direct chemical and/or osmotic irritation of the intima and mechanical distension from volume of drug injected (14). Prevention or reduction of pain can be achieved by running potassium chloride at an infusion rate below $20 \mathrm{mmol} /$ hour (15), injecting into large vein using a large bore cannula, adding a diluent (e.g., 0.9\% normal saline) during the infusion process, and applying an ice pack.

Previous studies have demonstrated the efficacy of lignocaine in alleviating pain during potassium chloride infusion both immediately and over an infusion time of up to 2 hours (14-16). However, the varying doses of lignocaine, small sample size, and subsequent reports of safety issues have limited the prevalent use of lignocaine in clinical practice. Safety issues have been attributed to the different formulations of lignocaine available and the extra step of preparation which led to an increased likelihood of medical error (17). Concerns have also been raised about the potential of lignocaine to mask phlebitis. More than a decade later, we embarked on this study to relook at the efficacy and safety related to the use of lignocaine in reducing pain during potassium chloride infusion. We hope that our findings will offer clinicians the option of a fairly simple intervention that will address the issue of pain experienced by patients during potassium chloride infusion.

\section{Materials and Methods}

\section{Setting}

This study was conducted in an Emergency Department (ED) in Singapore that sees about 135,000 patients a year.

\section{Design}

A prospective observational study of the effect of lignocaine on pain during potassium chloride infusion was recorded in a continuous quality improvement database from March 2015 to May 2016. Patients were recruited by consecutive sampling. They were included if the attending ED physician ordered intravenous potassium replacement with potassium chloride. Patients with a history of epilepsy, cardiac arrhythmia, and sensitivity to lignocaine were excluded from the study. Baseline characteristics of age, gender, concentration of serum potassium, duration of potassium chloride infusion, and concurrent run of normal saline were recorded prior to the initiation of lignocaine.
Potassium chloride was given as a premixed solution containing 10 $\mathrm{mmol}$ of potassium chloride in $100 \mathrm{~mL}$ of water for injection. This commercially available preparation was routinely used for potassium chloride infusion as per departmental protocol. Patients who received lignocaine were given a $3 \mathrm{~mL}$ bolus of $1 \%$ solution a minute before the start of the potassium chloride infusion. This intervention was unblinded to the patient and attending staff. Patients were put on cardiac monitoring during the period of intravenous replacement if they had received lignocaine.

All doctors and nurses working in the ED were briefed on the lignocaine protocol. As this practice was recommended but not a standard-of-care in the department, the decision to administer lignocaine to the patient was left to the discretion of the attending physician.

\section{Outcome}

The primary outcome was to determine the efficacy of lignocaine in reducing pain experienced during potassium chloride infusion. The qualitative occurrence of self-reported pain was tracked instead of a quantitative pain score during the potassium chloride infusion. This was chosen as a practical outcome measure, as any occurrence of pain would necessitate intervention from the attending staff to help the patient tolerate the infusion. These interventions were dependent on the attending physician and included applying ice pack, slowing down the initial infusion rate, and stopping the infusion due to patient's refusal. The secondary outcome was to determine the adverse events associated with intravenous lignocaine use for this purpose. Adverse events were defined as lignocaine toxicity, cardiac arrhythmia, masking of phlebitis, and medication error. Occurrence of any pain or adverse event was recorded by the attending staff and subsequently traced from review of the patients' case records.

\section{Sample size}

On the basis of the previous study by Lim et al. (14), the incidence of pain in patients who did not receive lignocaine was $85.7 \%$ compared with $28.6 \%$ when lignocaine was given. For a reduction of at least $50 \%$ in the occurrence of pain in patients who received lignocaine prior to the potassium chloride infusion, a sample size of 22 with equal numbers in each arm was required to power our study $(\alpha=0.05, \beta=0.2$, power $=0.8)$. To increase the robustness of the study, patients were enrolled in the study until the end of the quality improvement initiative that would recruit 50 patients in each arm of the study as determined a priori.

\section{Statistical analysis}

To control for selection bias in the assignment of subjects to treatment due to the baseline characteristics, stabilized inverse probability of treatment weights (IPTWs) were used in the analysis to estimate the efficacy of lignocaine on pain outcome. IPTWs were derived using weights on the basis of propensity scores to create a pseudo-sample in which the distribution of the measured baseline covariates was independent of the treatment assignment (18). Propensity scores of the subjects were estimated using the multivariable logistic regression where the status of treatment was regressed on the measured baseline covariates. Stabilized IPTWs will reduce variability due to instability in estimation that could be induced either by those treated subjects with low propensity scores or untreated subjects with high propensity scores $(18,19)$. Absolute standardized difference was calculated for each of the baseline covariates to assess the balance be- 
tween both groups and was compared before and after applying stabilized weights. An absolute standardized difference of less than 0.1 was used to indicate negligible difference in the mean or prevalence of a covariate between the treatment and control group.

Risk estimates and numbers needed to treat (NNT) were estimated using stabilized weights. Chi-square test was used to test for differences between categorical variables and two-sample t-test was used for continuous variables. All statistical analyses were performed using Statistical Package for the Social Sciences (IBM SPSS Statistics; Armonk, NY, USA) version 24.

\section{Results}

One hundred eligible patients were enrolled consecutively in the study to meet the sample size of 50 patients in the treatment group where lignocaine was given prior to the initiation of potassium chloride and another 50 patients in the control group with no lignocaine given.

The baseline demographics and clinical characteristics of the 100 patients are presented in Table 1. The average age of patients included in the study was $64.5( \pm 19.3)$ years. Majority of the patients were females (70\%). Eighty-one percent of all patients required more than 1 hour of potassium chloride infusion, and $79 \%$ of the patients required concurrent run of normal saline due to their presenting

Table 1. Patients' baseline demographics and clinical characteristics

\begin{tabular}{|l|c|}
\hline Variable & $\mathbf{n = 1 0 0}$ \\
\hline Age, mean ( \pm SD), years & $64.5( \pm 19.3)$ \\
\hline Gender (\%) & 30.0 \\
\hline Male & 70.0 \\
\hline Female & $2.7( \pm 0.4)$ \\
\hline Serum Potassium, mean ( \pm SD), mmol/L & 19.0 \\
\hline Infusion duration required (\%) & 81.0 \\
\hline$=1$ hour & \\
\hline$>1$ hour & 79.0 \\
\hline Concurrent Normal Saline (\%) & 21.0 \\
\hline Given & \\
\hline Not given &
\end{tabular}

medical conditions. The average serum potassium concentration of patients was $2.7( \pm 0.4) \mathrm{mmol} / \mathrm{L}$.

Prior to stabilized weights adjustment, all covariates, except gender, had an absolute standardized difference of more than 0.1 , with a maximum of 0.55 for the mean serum potassium concentration $(p=0.007$ ) (Table 2). On average, patients in the lignocaine group tend to be younger (mean age, 63.0 vs. 66.0 years) and had a higher level of serum potassium concentration ( $2.8 \mathrm{vs}$. $2.6 \mathrm{mmol} / \mathrm{L}$ ). The proportion of patients who required potassium chloride infusion of more than 1 hour was lower in the lignocaine group than in the control group (74.0\% vs. $86.0 \%$ ). More patients in the lignocaine group had concurrent run of normal saline solution during the potassium chloride infusion ( $84.0 \%$ vs. $74.0 \%)$. There was no absolute difference in the proportion of gender between the two groups.

After stabilized weights adjustment, the resultant sample size was 98, with 49 patients in each group. There were no significant differences in the baseline covariates between the two groups. The absolute standardized differences for all covariates in the weighted sample ranged from a maximum of 0.1 to a minimum of 0.03 . Balance of baseline covariates between the lignocaine and the control group was achieved in the stabilized weights adjusted sample.

The efficacy of lignocaine on pain was estimated on the basis of the weighted sample where the assignment of patients to lignocaine was independent on the patients' baseline characteristics. The proportion of patients experiencing pain was significantly higher in the control group where no lignocaine was given prior to the potassium chloride infusion (38.8\% vs. $16.3 \%, \mathrm{p}=0.013$ ) (Figure 1 ).

The absolute risk reduction was $22.5 \%$ and the relative risk of pain associated with lignocaine was 0.42 (95\% C.I., 0.20-0.87, p=0.013). In other words, the risk of experiencing pain for patients who were not assigned to the lignocaine group was 2.4 times the risk of those who were given lignocaine. The numbers needed to treat was 5 (95\% C.I., 3-19), suggesting that on average, for every five patients who were treated with lignocaine prior to the initiation of potassium chloride infusion, one will benefit from the treatment.

There were no adverse events associated with the lignocaine protocol used in this study, particularly the occurrence of cardiac arrhythmias, lignocaine toxicity, and medication errors.

Table 2. Characteristics of patients for the unweighted and weighted samples

\begin{tabular}{|c|c|c|c|c|c|c|}
\hline & \multicolumn{3}{|c|}{ Unweighted } & \multicolumn{3}{|c|}{ Weighted by Stabilized IPTWs } \\
\hline & $\begin{array}{c}\text { Lignocaine } \\
(n=50)\end{array}$ & $\begin{array}{l}\text { No Lignocaine } \\
(n=50)\end{array}$ & $\stackrel{p}{\mathbf{p}}$ & $\begin{array}{l}\text { Lignocaine } \\
(n=49)\end{array}$ & $\begin{array}{c}\text { No Lignocaine } \\
(n=49)\end{array}$ & $\stackrel{p}{\text { (Std Diff) }}$ \\
\hline Male & & & $(0.00)$ & & $14(28.6)$ & $(0.04)$ \\
\hline Age, mean $( \pm S D)$, years & $63.0( \pm 19.7)$ & $66.0( \pm 19.1)$ & $0.441(0.15)$ & $64.6( \pm 19.5)$ & $65.1( \pm 18.8)$ & $0.883(0.03)$ \\
\hline Infusion duration more than 1 hour, n (\%) & $38(76.0)$ & $43(86.0)$ & $0.202(0.26)$ & $38(77.6)$ & $40(81.6)$ & $0.616(0.10)$ \\
\hline Concurrent Normal Saline given, n (\%) & $42(84.0)$ & $37(74.0)$ & $0.220(0.25)$ & $39(79.6)$ & $38(77.6)$ & $0.806(0.05)$ \\
\hline
\end{tabular}




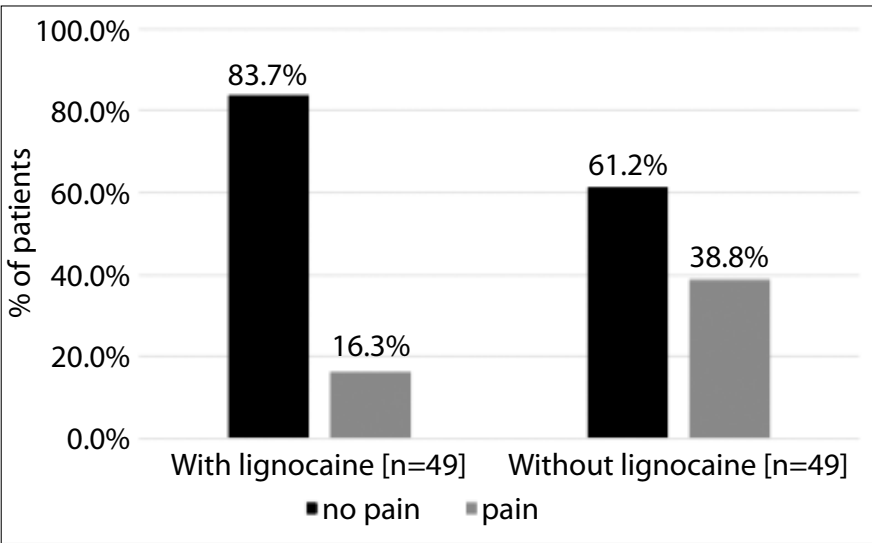

Figure 1. Proportion of patients experiencing pain during potassium chloride infusion

\section{Discussion}

\section{Pain control and lignocaine}

Several lignocaine regimens have been used in previous studies to reduce the pain during potassium chloride infusion. Lim et al. (14) used $3 \mathrm{~mL}$ of $1 \%$ (30 mg) lignocaine as a bolus 1 minute before starting a $20 \mathrm{mmol}$ potassium chloride diluted in $100 \mathrm{ml}$ dextrose $5 \%$ infusion which was administered over 2 hours. Morrill et al. (15) used 10 $\mathrm{mg}$ of lignocaine added to $10 \mathrm{mmol}$ of potassium chloride in $50 \mathrm{ml}$ of dextrose $5 \%$ which was ran over 1 hour. Pucino et al. (16) used 50 $\mathrm{mg}$ of lignocaine added to $20 \mathrm{mmol}$ of potassium chloride in $50 \mathrm{ml}$ of dextrose $5 \%$ which was administered over 2 hours. The preparation of potassium chloride used in our institution was $10 \mathrm{mmol}$ in $100 \mathrm{~mL}$ of water for injection, which was less concentrated than the solutions used in previous studies. Therefore, the incidence of pain in our patients without lignocaine was $38.8 \%$, lower than the $60 \%$ reported in literature. To ensure that the lignocaine dose we used was effective in controlling pain and had minimal risk for adverse event, we decided to use $3 \mathrm{~mL}$ of $1 \%$ lignocaine for in our study. Furthermore, Lim et al. (14) has shown that this dose of lignocaine was effective for pain control both immediately and throughout a 2-hour infusion period, attributable to the duration of action of lignocaine $\left[\mathrm{t}_{1 / 2}, 80-108 \mathrm{~min}\right.$ utes $(20,21)]$. With the increased in sample size, our study had shown that lignocaine was able to reduce to pain associated with potassium chloride infusion. At $\mathbf{\$} \$ 0.74$ per vial and a number needed to treat of 5 , this intervention was inexpensive, in addition to being safe at the study regimen.

We would want to highlight that the technique of slow intravenous push when administering the bolus lignocaine dose was important for anesthetizing the nerves fibers that supply the veins. A rapid bolus would deliver the drug systematically rather than allowing lignocaine to act locally and may render the lignocaine bolus ineffective.

\section{Intervention needed}

Application of ice pack was done more commonly in $71.4 \%$ patients as compared to slowing down of infusion rate in $25 \%$ patients who experienced pain. It was unlikely that the attending ED physician would agree to slow down the infusion rate as the first measure after making the decision to initiate intravenous replacement therapy for severe, symptomatic hypokalemia. Our study did not standardize the use of a large vein (e.g., cubital fossa) or a particular cannula size as in previous studies. By leaving this to the attending ED physicians, a pragmatic trial would demonstrate the efficacy of lignocaine in reducing pain during potassium chloride infusion.

\section{Adverse outcomes}

While there was genuine concern for potential adverse events from lignocaine use, this likelihood was low at the prescribed dose of 30 $\mathrm{mg}$. For an average $70 \mathrm{~kg}$ adult, this was $0.4 \mathrm{mg} / \mathrm{kg}$, way less than the toxic dose of $3 \mathrm{mg} / \mathrm{kg}$ for lignocaine where cardiac arrhythmias and other features of lignocaine toxicity ranging from circumoral numbness to seizures may occur. To address the concern of cardiac arrhythmias, our patients were put on cardiac monitoring throughout the period of infusion during the study. No arrhythmia was noted with this dosage of lignocaine. Therefore, the routine use of cardiac monitoring with lignocaine in our study's prescribed dose and use was not recommended. There were also no reports of features attributable to lignocaine toxicity. Phlebitis could be avoided by assessing the injection site before running the potassium chloride infusion with lignocaine.

Another reason contributing to the infrequent use of lignocaine to reduce pain from potassium chloride infusion was medical error arising from an extra step in preparation. Errors had resulted when staff used the wrong formulation due to the different concentrations and preparations of lignocaine currently available. This should not be a reason to deny patients a simple intervention which could be carried out by trained staff adhering to stringent preparation protocols, as with many other medications being used in any hospitals.

\section{Study limitations}

The main limitation of this observational study was the lack of randomization of patients into receiving lignocaine prior to potassium chloride infusion. However, we attempted to control for this by the use of stabilized weights in the statistical analysis. Nonetheless, unidentified confounders may still exist, affecting the results.

Another limitation was that absolute occurrence of pain was tracked instead of pain score. Therefore, for patients who experienced pain during the infusion, we were unable to compare the difference in pain scores between the two groups. This decision was based on an ideal outcome, as the pretreatment with lignocaine should alleviate all pain associated with the infusion, removing the need for intervention from the attending staff. The extent of pain score reduction with lignocaine during potassium chloride infusion and its effectiveness would need to be evaluated in a separate study.

\section{Conclusion}

The addition of lignocaine to intravenous potassium chloride replacement was useful in reducing pain during the infusion process. This would improve patient acceptance of the therapy and also reduce the need for additional intervention from the attending staff. In addition, we have proven that this extra step in preparation could be done safely and the addition of lignocaine was not associated with any adverse events as previously thought. Therefore, we recommend the routine use of $3 \mathrm{ml}$ of $1 \%$ lignocaine prior intravenous potassium chloride replacement as a new practice standard. 
Peer-review: Externally peer-reviewed.

Conflict of Interest: No conflict of interest was declared by the authors.

Financial Disclosure: The authors declared that this study has received no financial support.

\section{References}

1. Rose BD, Post TW. Hypokalemia. In: Clinical Physiology of Acid-Base and Electrolyte Disorders, 5th ed, Rose BD, Post TW (Eds), McGraw-Hill, New York 2001. p.836.

2. Comi G, Testa D, Cornelio F, Comola M, Canal N. Potassium depletion myopathy: a clinical and morphological study of six cases. Muscle Nerve 1985; 8: 17-21. [CrossRef]

3. Shintani S, Shiigai T, Tsukagoshi H. Marked hypokalemic rhabdomyolysis with myoglobinuria due to diuretic treatment. Eur Neurol 1991; 31: 3968. [CrossRef]

4. Dominic JA, Koch M, Guthrie GP Jr, Galla JH. Primary aldosteronism presenting as myoglobinuric acute renal failure. Arch Intern Med 1978; 138: 1433-4. [CrossRef]

5. Knochel JP. Neuromuscular manifestations of electrolyte disorders. Am J Med 1982; 72: 521-35. [CrossRef]

6. Mount DB. Causes of hypokalemia in adults. In: UpToDate, Post TW (Ed), UpToDate, Waltham, MA. (Accessed on August 5, 2016)

7. Cohn JN, Kowey PR, Whelton PK, Prisant LM. New guidelines for potassium replacement in clinical practice: a contemporary review by the $\mathrm{Na}$ tional Council on Potassium in Clinical Practice. Arch Intern Med 2000; 160: 2429-36. [CrossRef]

8. Kim GH, Han JS. Therapeutic approach to hypokalemia. Nephron 2002; 92(Suppl 1): 28. [CrossRef]

9. Villamil MF, Deland EC, Henney RP, Maloney JV Jr. Anion effects on cation movements during correction of potassium depletion. Am J Physiol 1975; 229: 161-6.
10. Kassirer JP, Schwartz WB. The response of normal man to selective depletion of hydrochloric acid. Factors in the genesis of persistent gastric alkalosis. Am J Med 1966; 40: 10-8. [CrossRef]

11. Kassirer JP, Berkman PM, Lawrenz DR, Schwartz WB. The critical role of chloride in the correction of hypokalemic alkalosis in man. Am J Med 1965; 38: 172-89. [CrossRef]

12. Stephen $M$, Loewenthal J, Wong J, Benn R. Complications of intravenous therapy. Med J Aust 1976; 2: 557-9.

13. Chan R, Armstrong D. Peripheral intravenous infusion of potassium chloride: effect of solution composition on infusion-site pain. Canadian $J$ Hosp Pharm 2004; 57: 27-31.

14. Lim ET, Khoo ST, Tweed WA. Efficacy of lignocaine in alleviating potassium chloride infusion pain. Anaesth Intensive Care 1992; 20 : 196-8.

15. Morrill GB, Katz MD. The use of lidocaine to reduce pain induced by potassium chloride infusion. J Intraven Nurs 1988; 11: 105-8.

16. Pucino F, Danielson BD, Carlson JD, Strommen GL, Walker PR, Beck CL, et al. Patient tolerance to intravenous potassium chloride with and without lidocaine. Drug Intell Clin Pharm 1988; 22: 676-9.

17. Grissinger M. Adding lidocaine to IV potassium infusions can cause safety problems. P T. 2008; 33: 70-5.

18. Peter CA. An Introduction to Propensity Score Methods for Reducing the Effects of Confounding in Observational Studies. Multivariate Behav Res 2011; 46: 399-424. [CrossRef]

19. Xu S, Ross C, Raebel MA, Shetterly S, Blanchette C, Smith D. Use of Stabilized Inverse Propensity Scores as Weights to Directly Estimate Relative Risk and Its Confidence Intervals. Value Health 2010; 13: 273-7. [CrossRef]

20. Boyes RN, Scott DB, Jebson PJ, Godman MJ, Julian DG. Pharmacokinetics of lidocaine in man. Clin Pharmacol Ther 1971; 12: 105-16. [CrossRef]

21. Rowland M, Thomson PD, Guichard A, Melmon KL. Disposition kinetics of lidocaine in normal subjects. Ann NY Acad Sci 1971; 179: 383-98. [CrossRef] 